Supplementary Information

\title{
Amine-Gold Linked Single-Molecule Circuits: Experiment and Theory
}

Su Ying Quek ${ }^{1}$, Latha Venkataraman ${ }^{2,3}$, Hyoung Joon Choi ${ }^{4}$, Steven G. Louie ${ }^{1,5}$, Mark S. Hybertsen $^{6,3}$, and J. B. Neaton ${ }^{1}$

${ }^{1}$ Molecular Foundry, Lawrence Berkeley National Laboratory, Berkeley, CA

${ }^{2}$ Department of Physics, Columbia University, New York, NY

${ }^{3}$ Center of Electron Transport in Nanostructures, Columbia University, NY, NY

${ }^{4}$ Department of Physics and IPAP, Yonsei University, Seoul, Korea

${ }^{5}$ Department of Physics, University of California, Berkeley, Berkeley, CA

${ }^{6}$ Center for Functional Nanomaterials, Brookhaven National Laboratory, Upton, NY

Content:

1. Experimental procedure

2. Details of data analysis procedure (Supplementary Figure 1)

3. Theoretical method

4. Details of theoretical results (Supplementary Table 1)

5. References 


\section{Experimental Procedure}

The conductance of single BDA molecules is measured by breaking Au point contacts in a 1,2,4-trichlorobenzene solution of the molecules using a modified scanning tunneling microscope as described in detail elsewhere ${ }^{1}$.

\section{Details of data analysis procedure}

The most probable conductance (peak position) and conductance spread (halfwidth at half-maximum) in the conductance histograms is obtained by performing a Lorentzian fit to the well-defined peak in the histogram. The spread is given as a percentage variation of the value of the position of the Lorentzian peak.

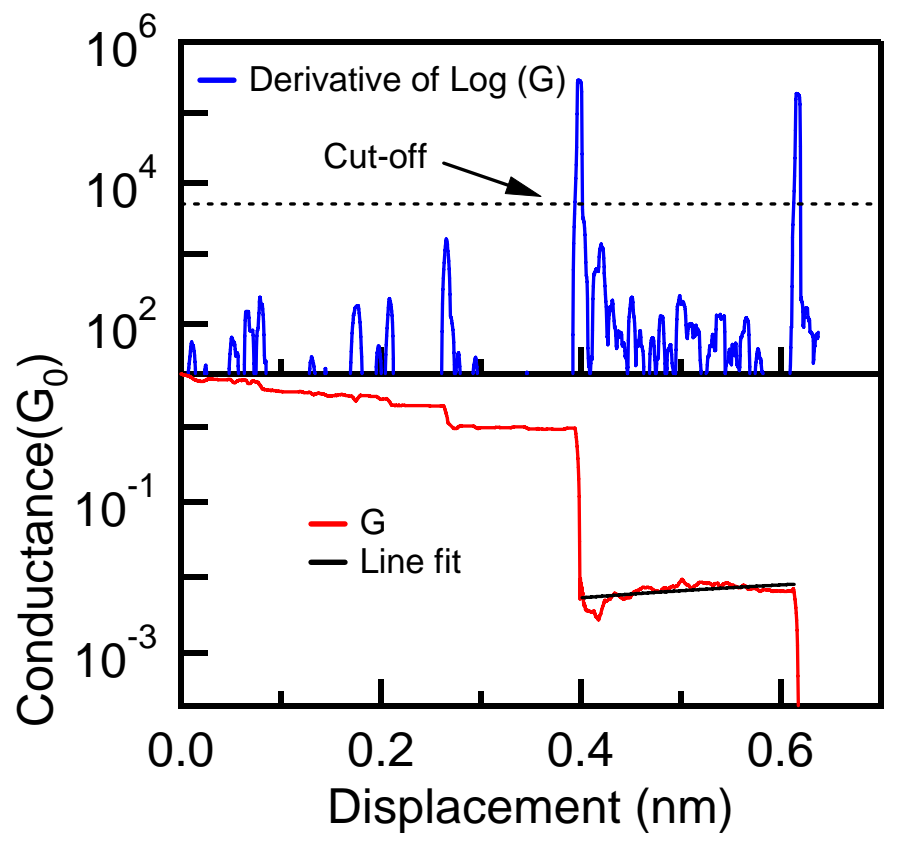

Supplementary Figure 1: A sample conductance trace (lower panel) and the derivative of the log of the traces (upper panel). A line fit to the molecular step is also shown.

The fractions of traces with steps, as well as the step length, step slope and step average conductance were determined from an automated sorting algorithm. For each measured 
trace, the derivative of the logarithm of the trace was computed. Traces with peaks in the derivative that crossed a threshold of 5000 were considered further. The average conductance from the raw data in the region between two successive peaks was computed. (See Supplementary Figure 1). Traces were considered to have molecular steps if this average conductance within this region was between $0.003 \mathrm{G}_{0}$ and $0.02 \mathrm{G}_{0}$ (the range of the black histogram peak shown in Fig. 1(b) of the main text) and if this region had more than 5 data points. Steps that had fewer than 5 data points, or equivalently those that were shorter than $0.007 \mathrm{~nm}$, were not included. A line was fit through this region and the slope and length of the step were determined from the fits.

\section{Theoretical Method}

Our calculations are performed in the framework of Density Functional Theory (DFT) as implemented in the SIESTA code ${ }^{2}$, within the generalized gradient approximation (GGA) ${ }^{3}$. An optimized single- $\varsigma$ basis set is used for the Au $d$ shell; all other orbitals are described by double- $\varsigma$ polarization basis sets. The calculated band structure for bulk Au agrees reasonably well with that from plane wave calculations, and the calculated Au bulk modulus, lattice constant, $\mathrm{Au}_{2}$ vibrational frequency and $\mathrm{Au}(111)$ work function agree reasonably well with experiment (respective errors of -7.5, +3.5, -2.6 and $+2.3 \%$ ). The DFT eigenvalues for a single BDA molecule bonded to a single Au atom at each amine group match those previously obtained using quantum chemistry calculations ${ }^{4}$.

Junctions are first constructed with 6-layer Au(111) slabs on either side of the BDA molecules, and with translational symmetry imposed along directions parallel to the Au surface. Our supercells have $16 \mathrm{Au}$ atoms per layer; we find that the junction density 
of states does not change significantly compared with that of $24 \mathrm{Au}$ atoms per layer. All atoms in the junction, except for those in the bottom 3 Au layers of each slab, are relaxed until forces are $<0.05 \mathrm{eV} / \AA ̊$. $\Gamma$-point sampling is sufficient for accurate geometry optimizations; BDA binding energies (reported in Supplementary Table 1) are obtained with a $2 \times 2 \times 1$ Monkhorst-Pack k-point mesh.

The conductance is obtained using a coherent elastic scattering state approach based on DFT. ${ }^{5,6}$ The BDA junction is divided into three regions: left bulk, center resistive region (4 Au layers on either side of molecule), and right bulk. The center region is chosen large enough for the Hartree potentials at its boundaries to smoothly match those of the bulk. The bulk regions are infinitely repeated away from the junction to simulate open boundary conditions. Energy- and $\mathbf{k}_{/ /}$-dependent scattering states are constructed with incoming and outgoing itinerant and evanescent states determined from the bulk Au complex band structure. Typical energy grid spacings used in this work are $10 \mathrm{meV}$. Care is taken to converge the conductance values with the $\mathbf{k}_{/ / \text {-mesh required to }}$ sample the two-dimensional Brillouin zone. An $8 \times 8$ Monkhorst-Pack $\mathbf{k}_{/ /}$-mesh is used for most junctions; in the (III',III') junctions, a $16 \times 16$ mesh is found to be necessary. Solving for the scattering states yields the transmission matrix t with elements $t_{n m}\left(E ; \mathbf{k}_{/ /}\right)$ for each incoming channel $\mathrm{n}$ and outgoing channel $\mathrm{m}$. The zero-bias conductance is then computed from the Landauer formula $\mathrm{G}=\mathrm{G}_{0} \Sigma_{\mathbf{k} / /} \operatorname{Tr}\left(\mathrm{t}^{\dagger} \mathrm{t}\right)$, where $\mathrm{t}$ is evaluated at the Fermi energy, $E_{F}$. Further details of our first-principles scattering-state approach can be found in References 5, 6. 
To simulate the stretching of junctions, we increase the distance between the left bulk and right bulk regions incrementally by $0.2 \AA$ from the equilibrium distance, and relax all atoms in the molecule, the Au contact, and the top three Au layers on each side. 


\section{Details of theoretical results}

\begin{tabular}{|c|c|c|c|c|c|c|c|}
\hline Junction & $n$ & $\mathrm{BE}(\mathrm{eV})$ & $\mathrm{G}\left(\mathrm{G}_{0}\right)$ & $\overline{E_{r}(e V)}$ & $\Gamma(\mathrm{eV})$ & $\mathrm{d}(\mathrm{N}-\mathrm{Au})(\AA)$ & $\theta\left(^{\circ}\right)$ \\
\hline (III', III') & 6 & 0.90 & 0.0470 & -1.20 & 0.37 & $(2.51,2.51)$ & $(130,130)$ \\
\hline$\left(\right.$ III', III') $_{c i s}$ & 6 & 0.89 & 0.0390 & -1.23 & 0.36 & $(2.49,2.49)$ & $(128,128)$ \\
\hline$\left(\right.$ III', III') $_{31^{\circ}}$ & 6 & 0.87 & 0.0601 & -1.07 & 0.38 & $(2.55,2.55)$ & $(130,130)$ \\
\hline (III', III') $)_{56^{\circ}}$ & 6 & 0.83 & 0.0318 & -1.23 & 0.34 & $(2.44,2.44)$ & $(112,112)$ \\
\hline (IV', IV') & 6 & 0.91 & 0.0411 & -1.27 & 0.45 & $(2.50,2.50)$ & $(129,129)$ \\
\hline (III', I) & 6 & 1.02 & 0.0371 & -1.20 & 0.42 & $(2.51,2.42)$ & $(128,127)$ \\
\hline$\left(\right.$ III', I I $_{31^{\circ}}$ & 6 & 1.05 & 0.0453 & -1.09 & 0.42 & $(2.53,2.45)$ & $(130,129)$ \\
\hline$(\mathrm{I}, \mathrm{I})$ & 6 & 1.19 & 0.0471 & -1.13 & 0.48 & $(2.38,2.38)$ & $(122,122)$ \\
\hline$(\mathrm{I}, \mathrm{I})_{40^{\circ}}$ & 6 & 1.30 & 0.0489 & -1.11 & 0.49 & $(2.38,2.38)$ & $(121,121)$ \\
\hline$(\mathrm{II}, \mathrm{I})$ & 7 & 1.22 & 0.0438 & -1.19 & 0.50 & $(2.41,2.40)$ & $(124,123)$ \\
\hline (II, II) & 8 & 1.18 & 0.0479 & -1.19 & 0.52 & $(2.45,2.45)$ & $(127,127)$ \\
\hline (III, I) & 8 & 1.22 & 0.0460 & -1.16 & 0.51 & $(2.42,2.40)$ & $(123,123)$ \\
\hline 'side' $10^{\circ}$ & 9 & 1.08 & 0.0416 & -1.09 & 0.48 & $(2.47,2.43)$ & $(121,123)$ \\
\hline (III, III) & 10 & 1.18 & 0.0537 & -1.13 & 0.56 & $(2.46,2.46)$ & $(125,125)$ \\
\hline$(\mathrm{IV}, \mathrm{IV})$ & 12 & 0.96 & 0.0626 & -0.94 & 0.49 & $(2.49,2.49)$ & $(122,122)$ \\
\hline
\end{tabular}

Supplementary Table 1: Binding energies (BE), conductances (G), resonance peak

positions $\left(\mathrm{E}_{\mathrm{r}}\right)$ and widths $(\Gamma)$ of Lorentzian fits, as well as $\mathrm{N}-\mathrm{Au}$ bond lengths $(\mathrm{d}(\mathrm{N}-\mathrm{Au}))$

and Au-N-C angles $(\theta)$ of the 15 junctions. $n$ refers to the total number of Au nearest

neighbors at the two Au binding sites, as defined in the main text. BEs are taken with

respect to the relaxed, isolated motifs on Au slabs and BDA; a more positive BE

corresponds to stronger adsorption. For the 'side' junction, $\mathrm{d}(\mathrm{N}-\mathrm{Au})$ and $\theta$ are given in 
sequence for the (III') and (I) contacts. For other junctions, this order follows that given in the name of the junction (e.g. in the (III, I) junction, the values of d(N-Au) are $2.42 \AA$ at contact (III) and $2.40 \AA$ at contact (I)). Unless otherwise labeled, all junctions have an $\sim 0^{\circ}$ angle between the $\mathrm{N}-\mathrm{N}$ axis and the surface normal, and a trans binding configuration (Au binding sites on opposite sides of molecular plane).

Supplementary Table 1 tabulates key physical quantities for the 15 junctions considered here. As described in the main text, the calculated conductance has a mean of $0.046 \mathrm{G}_{0}$, and ranges from $0.032(-31.1 \%)$ to $0.062(+35.4 \%) \mathrm{G}_{0}$. The BDA binding energies (BE) range from $0.9 \mathrm{eV}$ for (III', III') and (IV', IV') junctions to 1.0, 1.2 and $1.3 \mathrm{eV}$ for (IV, IV), (III, III)/(II, II) and (I, I) junctions respectively. The BEs in asymmetric junctions reflect the different binding strengths at the two contact structures. For a given set of motifs, other variations in geometry do not change the BE by more than $0.1 \mathrm{eV}$. The $\mathrm{N}-\mathrm{Au}$ bond lengths, $\mathrm{d}(\mathrm{N}-\mathrm{Au})$, range from 2.38 to $2.53 \AA$. The $\mathrm{N}-\mathrm{Au}$ bond lengths reflect the respective binding strengths at the different tip structures, with bonding at the (I) structure resulting in the shortest $\mathrm{N}-\mathrm{Au}$ bond lengths, and bonding at the (III') structure the longest. The transmission resonance width $\Gamma$, determined from a Lorentzian fit to the right tail of the resonance, ranges from 0.34 to $0.56 \mathrm{eV}$. $\Gamma$ tends to increase with increasing $n$ (the total number of Au nearest neighbours at the two Au binding sites). For $n=6$, $\Gamma$ increases with the number of second-nearest neighbors at the binding sites, with (I) structures resulting in larger $\Gamma$, and (III') structures in smaller $\Gamma$. The correlation between $\Gamma$ and the binding site coordination suggest that the width of the transmission resonances is determined by the degree of coupling to bulk $\mathrm{Au}$, rather than by the BDA BEs. The Au-N-C angle $\theta$ ranges from $112^{\circ}$ to $130^{\circ}$. This variation in $\theta$ 
helps accommodate the range of molecular configurations described here. The resonance energies range from -1.27 to $-0.94 \mathrm{eV}$. This relatively small percentage variation in resonance energy is reflected in the similar Mulliken charge populations (4.52-4.54 e) on $\mathrm{N}$ in the various contact structures.

\section{References}

1. Venkataraman, L.; Klare, J. E.; Tam, I. W.; Nuckolls, C.; Hybertsen, M. S.; Steigerwald, M. L., Nano Lett. 2006, 6, 458-462.

2. $\quad$ Soler, J. M.; Artacho, E.; Gale, J. D.; Garcia, A.; Junquera, J.; Ordejon, P.; Sanchez-Portal, D., Journal of Physics-Condensed Matter 2002, 14, $2745-2779$.

3. $\quad$ Perdew, J. P.; Burke, K.; Ernzerhof, M., Phys. Rev. Lett. 1996, 77, 3865-3868.

4. Venkataraman, L.; Klare, J. E.; Nuckolls, C.; Hybertsen, M. S.; Steigerwald, M. L., Nature 2006, 442, 904-907.

5. Neaton, J. B.; Khoo, K. H.; Spataru, C. D.; Louie, S. G., Computer Physics Communications 2005, 169, 1-8.

6. Choi, H. J.; Cohen, M. L.; Louie, S. G., Phys. Rev. B 2007, submitted. 